\title{
The Use of Maximum Light in Cepheid Distance Estimators
}

\section{S.M. Kanbur}

Department of Physics and Astronomy, University of Glasgow

\author{
M.A. Hendry \\ Astronomy Centre, University of Sussex
}

\begin{abstract}
Cepheid PLC relations are usually constructed using mean light. We show that the use of maximum and mean light leads to a distance estimator for the LMC with $30 \%$ less scatter than existing relations.
\end{abstract}

\section{Introduction}

Simon, Kanbur \& Mihalas (1993) showed that the range of Galactic Cepheid temperatures at maximum light is about half that at mean light. In order to use this in constructing Cepheid PLC relations, Kanbur \& Hendry (1995, hereafter $\mathrm{KH}$ ) derived the following relation between mean and maximum light for a Cepheid,

$$
\log P-\alpha M_{\mathrm{v}}+\beta M_{\mathrm{vmax}}+\gamma(B-V)_{\max }=\text { constant, }
$$

where $\alpha, \beta, \gamma$ are constants and the other symbols have their usual meanings. Using the well known relation between apparent and absolute magnitude, we obtain an estimate of distance as

$$
\widehat{\log D}=0.2\left(m_{\mathrm{vmax}}-\hat{M}_{\mathrm{vmax}}-25\right),
$$

which gives the distance in Mpc. Our model for $\hat{M}_{\mathrm{vmax}}$ is given by a modified form of equation (1),

$$
\hat{M}_{\mathrm{vmax}}=a+b \log P+c(B-V)_{\max }+d\left(M_{\mathrm{vmean}}-<M_{\mathrm{vmean}}>\right),
$$

where all terms on the right hand side are directly observable and in particular the final term is just numerically equal to $m_{\mathrm{vmean}}-<m_{\mathrm{vmean}}>$, the mean apparent magnitude minus the sample average of the mean magnitudes of a group of equidistant Cepheids. We can also formulate a PLC(max) estimator by using

$$
\hat{M}_{\mathrm{vmax}}=a+b \log P+c(B-V)_{\max },
$$

in equation 2 , the justification again coming from equation (1). 


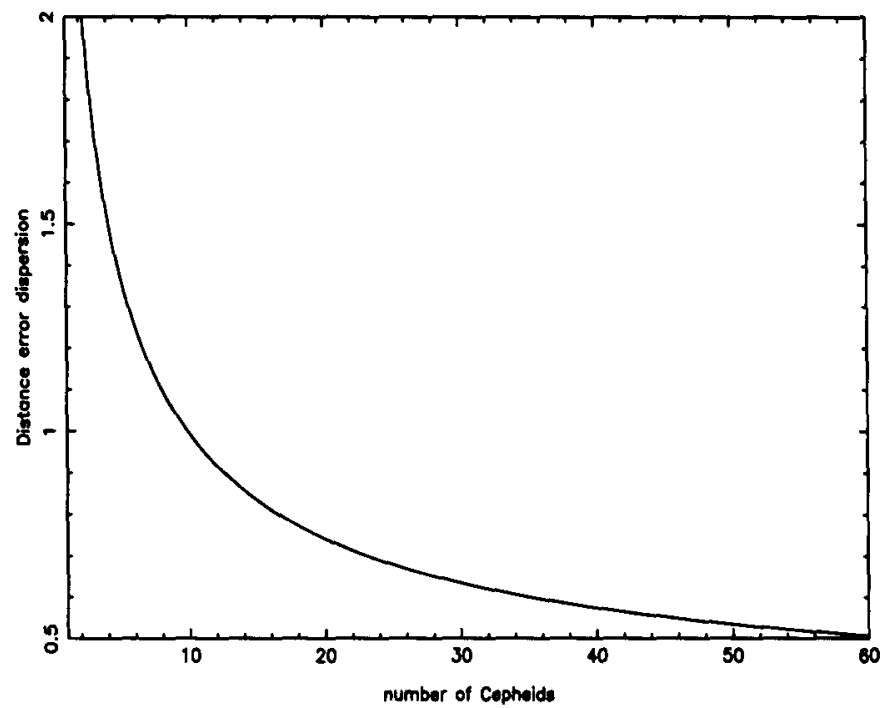

Figure 1. Rms percentage distance error derived from equation 3 divided by the percentage error of that derived from equation 4 as a function of $n$, the number of distant Cepheids.

\section{Results and discussions}

We have tested the distance estimators given by equations 2 and 4 using data for the LMC obtained by Martin, Warren \& Feast (1979). The statistical techniques used do take account of correlations between the variables and are described in $\mathrm{KH}$ and Hendry \& Simmons (1994). We found that the PLC(max) estimator given by equation 4 has about 10 percent less scatter than a PLC estimator using mean light. In Figure 1, we plot the rms percentage error derived from equation 3 divided by the percentage distance error of the PLC relation at maximum light - equation 4. It can be seen that when the number of Cepheids in the distant sample exceeds about 30 , we obtain a significant reduction in scatter when using equation 3 . These results may have interesting consequences for the cosmic distance scale.

\section{References}

Hendry, M.A., \& Simmons, J.F.L. 1994, ApJ, 435, 515

Kanbur, S.M., \& Hendry, M.A. 1995, A\&A, submitted

Martin, W.L., Warren, P.R., \& Feast, M.W. 1979, MNRAS, 188, 139

Simon, N.R., Kanbur, S.M., \& Mihalas, D. 1993, ApJ, 414, 310 